\title{
Full surface strain measurement using shearography
}

\author{
Roger M. Groves, Stephen W. James* and Ralph P. Tatam \\ Centre for Photonics and Optical Engineering, School of Engineering, Cranfield University
}

\begin{abstract}
Shearography is a full-field optical technique usually used for the determination of surface strain. Correlation of interferometric speckle patterns recorded before and after the object deformation yields fringes sensitive to displacement gradient, from which the surface strain can be calculated. A full analysis of the surface strain requires the measurement of six displacement gradient components, using three illumination directions and two directions of applied shear. Additionally shearography may be used to measure surface slope by correlation of interferograms obtained before and after a source displacement to yield fringes sensitive to surface slope. Integration of the slope yields the object shape. In this paper shearography is used to measure the six components of displacement gradient of a gas main pipe under pressure, the surface slope of the pipe and the shape of the pipe. The object slope and shape are used to correct the displacement gradient measurements for variation in sensitivity vector across the object surface and for sensitivity variations due to the dependence of the applied shear upon the local slope of the object surface. A coordinate transformation, incorporating the object shape information, is used to obtain the in-plane and out-of-plane displacement gradients relative to the local profile of the surface.
\end{abstract}

Keywords: strain measurement, non-destructive testing, shearography, shape measurement

\section{INTRODUCTION}

Speckle shearing interferometry, often termed shearography ${ }^{1,2}$, is a full-field non-contact optical technique, usually used for the qualitative measurement of changes in the displacement gradient caused by surface and subsurface defects. A speckle pattern is formed by illuminating an optically rough surface with an expanded laser beam. In shearography this speckle pattern is optically mixed with an identical but displaced, or sheared, speckle pattern using a shearing device, and viewed through a lens, forming a speckle interferogram at the camera surface. The shearing device commonly used for shearography is a Michelson interferometer with a tilt applied to one of the mirrors ${ }^{1}$. Correlating speckle interferograms obtained before and after object deformation yields correlation fringes sensitive to displacement gradient in the shear direction. Phase-stepping techniques ${ }^{3}$ are often used to extract the phase information, and therefore the displacement gradient information, from the speckle interferograms.

Shearography is able to measure six orthogonal components of displacement gradient allowing full characterisation of the surface strain components. To determine these six components, measurements are performed with three different directions of the sensitivity vector, which is the bisector of the illumination and viewing directions, and with two orthogonal directions of applied shear. The magnitude of the applied shear controls the sensitivity of the displacement gradient measurement. The measured components of displacement gradient are non-orthogonal and the orthogonal displacement gradient components are determined by a coordinate transformation.

An object that is curved, or has angled surfaces relative to the camera image plane, has a different magnitude of applied shear to that measured in the image plane. This variation in the applied shear relative to the measured magnitude of applied shear may be corrected for by considering the surface slope of the object in the shear direction. A knowledge of the object shape allows the sensitivity vector to be locally determined across the object surface when performing the coordinate transformation. It is desirable to calculate the displacement gradient components, relative to the object surface profile, and this may also be performed using a knowledge of the object shape.

\footnotetext{
* s.w.james@ cranfield.ac.uk; phone 01234 754623; fax 01234 752452; http://www.cranfield.ac.uk/sme/cpoe; Optical Sensors Group, Centre for Photonics and Optical Engineering, School of Engineering, Cranfield University, Cranfield, Bedford, MK43 0AL, UK.
} 
Surface slope measurement may be performed using shearography. Correlating speckle interferograms recorded before and after a change in the illumination conditions ${ }^{4,5,6}$ generates correlation fringes sensitive to surface slope in the shear direction. The shape of the object is determined by integrating the surface slope.

In this paper a welded joint in a gas main pipe is investigated. Six components of displacement gradient are measured using three illumination directions, multiplexed in the time domain, a single camera and shearing head, and two orthogonal directions of applied shear. The surface slope is measured using a source displacement shearography technique ${ }^{5}$. The surface slope is integrated to obtain the shape of the object. A correction to the variation in applied shear across the object surface is made using surface slope information. The object shape is used to calculate local sensitivity vectors across the object surface, for each measurement channel. A coordinate transformation, using object shape information, is performed on the measured displacement gradient components to obtain the in-plane and out-ofplane displacement gradients across the surface of the object.

\section{THEORY}

\subsection{Multi-component strain measurement using shearography}

Shearography is a full field optical technique that can be used for the measurement of displacement gradient. A speckle pattern is formed by illuminating an object, which has an optically rough surface, by an expanded laser beam. The speckle pattern is optically mixed with an identical but displaced, or sheared, speckle pattern in a shearing interferometer and viewed through a lens forming an interferometric speckle pattern which can be recorded by the camera. Correlating speckle patterns obtained before and after object deformation yields correlation fringes sensitive to displacement gradient. Phase-stepping techniques ${ }^{3}$ can be used to extract the phase information, and therefore the displacement gradient, from the speckle interferograms.

The component of displacement gradient measured by shearography is determined by the sensitivity vector, which is the bisector of the illumination direction and the viewing direction, and by the direction of the applied shear. The out-ofplane components of displacement gradient can be measured directly by utilising illumination and viewing normal to the object surface. To measure the in-plane components of displacement gradient requires the measurement of displacement gradient from two directions of sensitivity vector such that each of the measured components of displacement gradient contains a contribution from the out-of-plane and the in-plane components. The in-plane and outof-plane components may then be calculated. If the illumination and viewing geometry is chosen to give opposing directions of sensitivity vector a subtraction can be used to remove the out-of-plane displacement gradient component. The second orthogonal in-plane displacement gradient component is determined by changing the illumination and viewing geometry.

A measurement of all the three displacement gradient components, the out-of-plane and two in-plane components, can be performed using either a single illumination direction and three viewing directions or a single viewing direction and three illumination directions. The latter is employed in this paper. A coordinate transformation is used to transform from the measured displacement gradient components to the out-of-plane and two in-plane components.

The optimum illumination geometry consists of three optical sources at three of the four corners of a square and with a minimum angle from the normal to the object surface of $11^{\circ}$. This is shown in Figure 1. The optimal positioning of the optical sources relative to the camera and the object is important to minimise errors introduced in performing the coordinate transformation.

The applied shear is determined by the direction and magnitude of the tilt applied to the shearing mirror in the shearing interferometer. Two orthogonal directions of applied shear, in conjunction with the three different illumination directions, are required to obtain the six components of displacement gradient required to fully characterise the surface strain. The magnitude of applied shear controls the measurement sensitivity and is typically of the order of 5 to $10 \mathrm{~mm}$. 


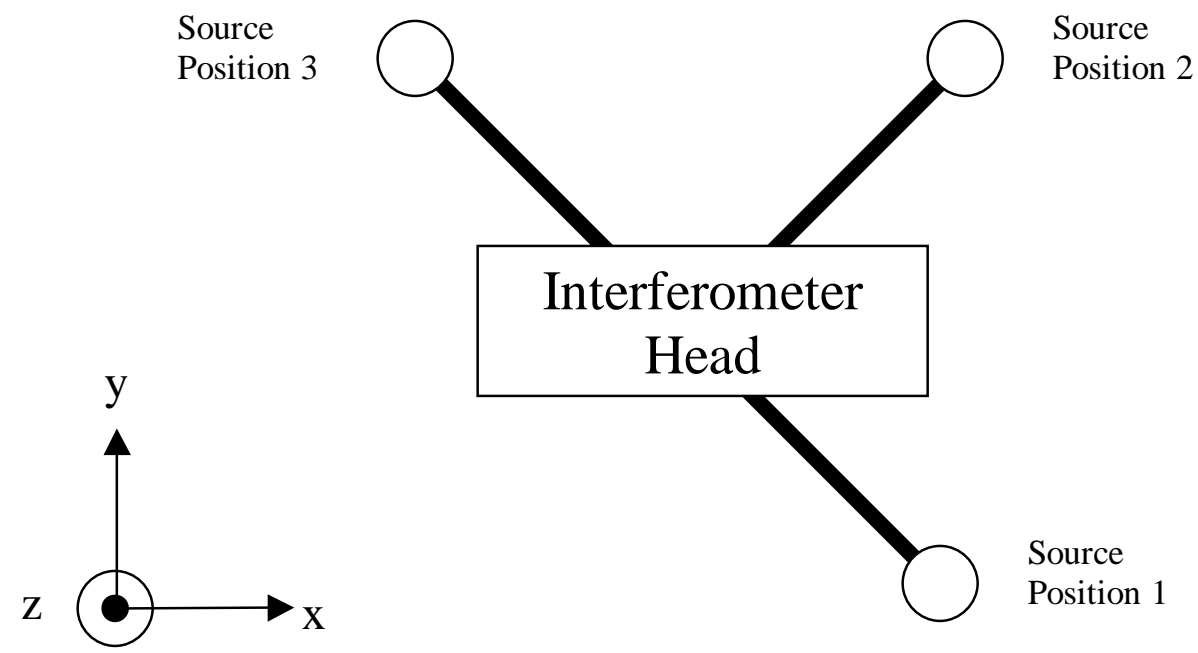

Figure 1 Positions of the optical sources relative to the interferometer head, viewed from the object position. The coordinate system used in this paper is shown, $\mathrm{x}$ and $\mathrm{y}$ are in the plane of the paper and $\mathrm{z}$ is normal to the plane of the paper.

The surface strain is determined from the six components of displacement gradient. Shearography is able to measure all the surface strain components but is not able to measure the bulk strain components, $\delta \mathrm{u} / \delta \mathrm{z}, \delta \mathrm{v} / \delta \mathrm{z}$ and $\delta \mathrm{w} / \delta \mathrm{z}$. The strain tensor, $\mathrm{S}$, is given by:

$$
S=\left[\begin{array}{ccc}
\frac{\partial u}{\partial x} & \frac{1}{2}\left(\frac{\partial u}{\partial y}+\frac{\partial v}{\partial x}\right) & \frac{1}{2}\left(\frac{\partial u}{\partial z}+\frac{\partial w}{\partial x}\right) \\
\frac{1}{2}\left(\frac{\partial v}{\partial x}+\frac{\partial u}{\partial y}\right) & \frac{\partial v}{\partial y} & \frac{1}{2}\left(\frac{\partial v}{\partial z}+\frac{\partial w}{\partial y}\right) \\
\frac{1}{2}\left(\frac{\partial w}{\partial x}+\frac{\partial u}{\partial z}\right) & \frac{1}{2}\left(\frac{\partial w}{\partial y}+\frac{\partial v}{\partial z}\right) & \frac{\partial w}{\partial z}
\end{array}\right]
$$

\subsection{Slope and shape measurement using shearography}

Shearography can be used to perform surface slope measurement via a source displacement technique ${ }^{5}$, from which the object shape can be determined. The technique is similar to that for measurement of displacement gradient. A reference interferometric speckle pattern is recorded. The optical source is displaced and a second interferogram is recorded. Correlating these two recorded interferograms yields correlation fringes sensitive to the surface slope of the object in the shear direction. Phase-stepping techniques ${ }^{3}$ can be used to extract the phase information from the correlation fringes. To fully characterise the surface slope measurements are made using two orthogonal directions of applied shear, to yield surface slope measurements in two orthogonal directions.

The nature of the fringes obtained using the source displacement technique in shearography is dependent on the direction of the source displacement and carrier fringes, slope sensitive fringes of a mixture of carrier and slope sensitive fringes can be generated. Carrier fringes are formed by a displacement to the optical along the source to object optical axis $^{8}$. A source displacement in an orthogonal direction generates predominantly slope fringes ${ }^{5}$. 
The surface slope sensitivity of correlation fringes generated in this way has previously been determined mathematically ${ }^{5}$. The relationship between the different system parameters and the sensitivity of the fringes to the surface slope of the object were investigated numerically and linear and non-linear relationships were identified. The source and illumination geometry, the direction of source displacement and the position in the camera field of view have complex non-linear relationships to the sensitivity of the fringes to object slope. For most practical experimental conditions the optical wavelength, the magnitude of source direction and the magnitude of applied shear have a linear relationship to the sensitivity of the slope sensitive fringes. The sensitivity vector must be inclined, with respect to the normal to the object surface, in the direction of the source displacement to give fringes sensitive to surface slope. The phase change $\phi_{\mathrm{S}}(\mathrm{x}, \mathrm{y})$ at a point $(\mathrm{x}, \mathrm{y})$ on the object surface is given by:

$$
\phi_{S}(x, y)=\frac{D V d x}{\lambda}\left(K \frac{d z}{d x}(x, y)+C(x, y)\right)
$$

where DV is the magnitude of a source displacement, predominantly in the $y$ direction, $d x$ is the applied shear, $\lambda$ is the optical wavelength, $K$ is the slope sensitivity constant, $d z / d x(x, y)$ is the surface slope at point $(x, y)$ and $C(x, y)$ is the zero slope constant. The slope sensitivity constant is calculated numerically using a model of the correlation fringe formation.

The zero slope constant is determined experimentally using a flat plate test object. The flat plate object replaces the object under investigation, with the orientation of the flat plate defining the plane of zero slope. The slope fringe formation procedure is repeated for the flat plate, using an identical direction and magnitude of source displacement, and after image processing values of $\mathrm{C}(\mathrm{x}, \mathrm{y})$ are determined across the field of view. These values of $\mathrm{C}(\mathrm{x}, \mathrm{y})$ can be subtracted from the slope, determined from the object under investigation, to remove any influence on these slope fringes from the change in sensitivity vector across the field of view. The scaling of the slope obtained is determined by using Equation 2.

\subsection{Strain measurement utilising slope and shape information}

The slope and shape information can be used to correct the displacement gradient measurements for slope and shape dependent measurement errors and can also provide a surface onto which the strain measurements can be mapped. Corrections are made for the applied shear, and therefore the displacement gradient sensitivity, varying across a curved surface and for the change in sensitivity vector across the object surface.

The applied shear is usually measured in the plane of the camera image. For a curved surface, or surface inclined with respect to the plane of the camera image, the applied shear on the object surface is larger, as the measured applied shear is stretched over a larger distance on the object surface. This error can be corrected using a knowledge of the object slope and simple geometry. The corrected shear, $\mathrm{dx}_{\mathrm{C}}$, is calculated using:

$$
d x_{C}=d x_{M} \sqrt{\left(\left(\frac{d z}{d x}\right)^{2}+1^{2}\right)}
$$

where $\mathrm{dx}_{\mathrm{M}}$ is the measured applied shear and $\mathrm{dz} / \mathrm{dx}$ is the surface slope in the $\mathrm{x}$ direction.

The error due to the variation in the sensitivity vector across the object surface can be corrected by calculating individual sensitivity vectors for points across the object surface using a knowledge of system geometry. A knowledge of the field of view and the shape of the object permits the location of the object surface in three-dimensional space, relative to a reference point on the object surface. The illumination positions and the viewing position relative to this reference point are measured. From this information the local sensitivity vector, for each of the three illumination directions, can be determined across the object surface.

A knowledge of the shape of the object also allows the surface strain components to be mapped onto the object surface. The local directions of the two in-plane and one out-of-plane vectors are determined using the information of the object shape and the measured displacement gradient components are transformed into this coordinate system at that point on 
the object surface. The calculation of the directions of the in-plane and out-of-plane components and the coordinate transformation are repeated across the object surface.

\section{EXPERIMENTAL}

The Cranfield multi-component shearography system ${ }^{7}$ was modified to incorporate a method of performing source displacement allowing both the displacement gradient measurements and the surface slope and shape measurements to be performed using the same system.

The multi-component shearography system utilised three illumination directions and one interferometer head to perform the measurements. Three fibre Bragg grating stabilised laser diodes $(810 \mathrm{~nm}, 100 \mathrm{~mW}$ optical power), with fibre optic beam delivery to the source position were used to illuminate the object. The Bragg gratings stabilised the output wavelength of the laser diodes when the laser injection current was modulated and the fibre optic beam delivery provided spatial filtering and a flexible beam delivery to the source position for the surface slope measurement channel. Lenses at the distal end of the fibres allowed an adjustment to the expansion of the beams illuminating the object. The source positions were nominally located at $1 .(0.2 \mathrm{~m},-0.2 \mathrm{~m}, 0.3 \mathrm{~m}), 2 .(0.2 \mathrm{~m}, 0.2 \mathrm{~m}, 0.3 \mathrm{~m})$ and $3 .(-0.2 \mathrm{~m}, 0.2 \mathrm{~m}, 0.3$ $\mathrm{m}$ ), measured from the centre of the field of view in the object plane, at three of the four corners of a square as shown previously in Figure 1. The optimum illumination geometry was determined previously ${ }^{7}$. Source position 3 was fitted with a translation stage at the end of the fibre allowing source displacement in three orthogonal directions. This channel was used to perform the surface slope and shape measurements.

The interferometer head was composed of a Michelson interferometer, camera lens and camera. The Michelson interferometer consisted of a beamsplitter, a shearing mirror that could be adjusted to give different magnitudes and directions of applied shear and a reference mirror, which was fitted with a piezoelectric transducer (PZT) allowing phase-stepping to be performed. The camera, an area scan CCD camera (Dalsa CA-D4-0512A, $75 \mathrm{~Hz}$ frame rate, 512 by 512 pixels), in conjunction with a PCI bus frame transfer card (Roadrunner) was used to record the speckle interferograms. A PC controlled the modulation of the laser diode injection currents, the phase-stepping and the image capture using a program written in $\mathrm{LABVIEW}^{\mathrm{TM}}$.

\subsection{Multi-component displacement gradient measurement}

To fully characterise the surface strain required the measurement of six components of displacement gradient. The six components were measured using three illumination directions and two orthogonal directions of applied shear. Figure 2 shows the experimental layout.

Initially the shear was applied in the $\mathrm{x}$ direction. The object was sequentially illuminated from the three illumination directions by modulating the Bragg grating stabilised laser diode injection currents between below threshold and the operating current, in synchronisation with the recording of reference camera frames. After deformation of the object the laser diode pulsing sequence was repeated, but with three phase-stepped signal frames recorded from each channel. The camera frames from an individual channel were processed using a phase-stepping algorithm to yield an unwrapped phase map and unwrapped using commercial software. This process was repeated for the three channels to yield three measured displacement gradient components. The shear was then applied in the y direction and the recording of reference and phase-stepped signal frames before and after object deformation, respectively, was repeated to yield the other three displacement gradient components. 


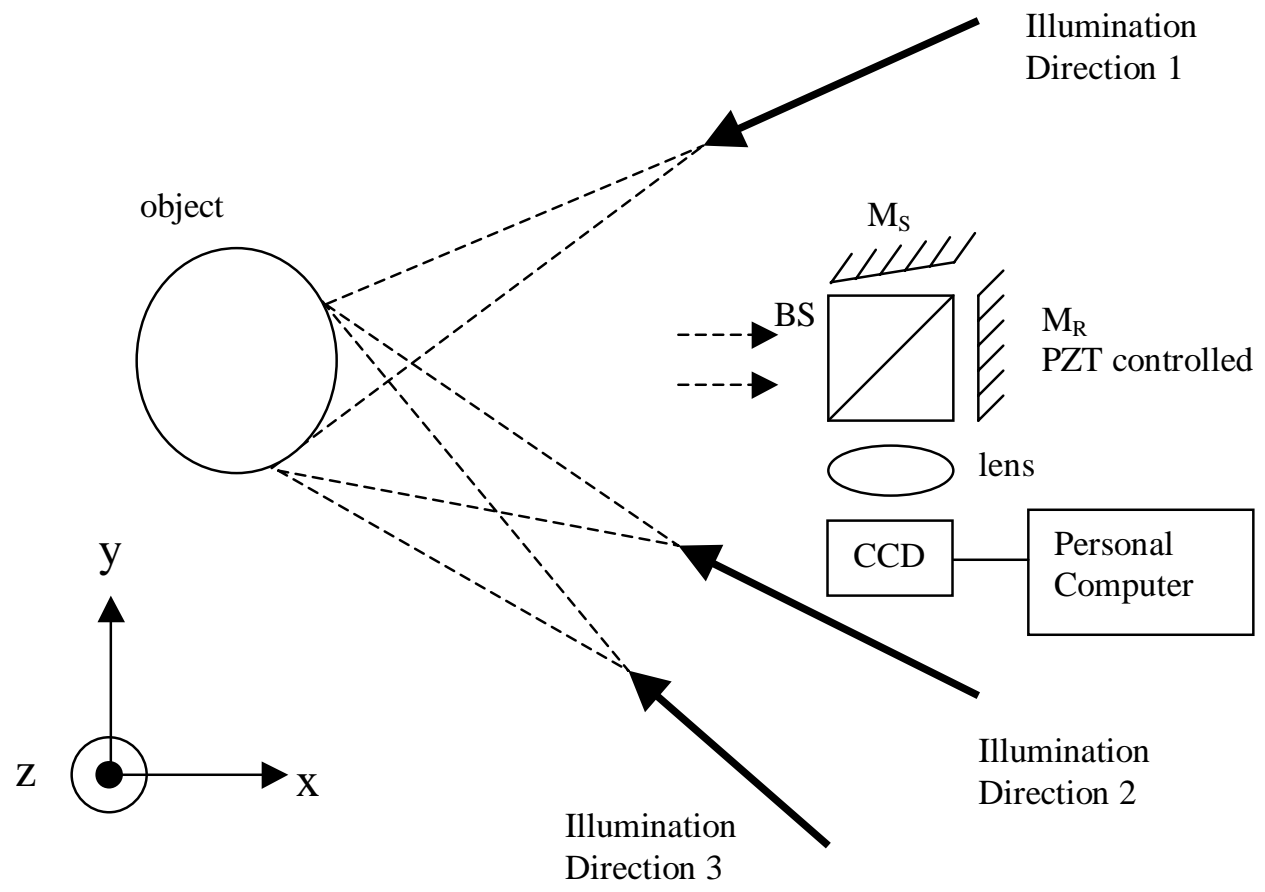

Figure 2 Experimental layout for displacement gradient measurement. Laser diodes, using single mode optical fibres for beam delivery, are used to illuminate the object from three directions. The shearing head, consists of beamsplitter, $\mathrm{BS}$; reference mirror controlled by a piezoelectric transducer, $\mathrm{M}_{\mathrm{R}}$; Shearing mirror, $\mathrm{M}_{\mathrm{S}}$; a lens and a CCD camera.

\subsection{Slope and shape measurement}

The experimental layout to perform surface slope measurement is shown in Figure 3. Illumination of the object was from source position 3. A reference frame was recorded first. The source was then displaced in the $\mathrm{v}$ direction by a magnitude of DV of the order of hundreds of microns and three phase-stepped signal frames were recorded. The $\mathrm{v}$ direction is orthogonal to the source to object optical axis and is predominantly in the y (vertical) direction. The reference and signal frames were combined using a phase-stepping algorithm to yield an unwrapped phase map. This was unwrapped using commercially available software. The object was replaced by a flat plate, with the normal to the surface in the direction of the interferometer head. The process of capturing a reference frame, displacing the source in the DV direction, recording three phase-stepped frames and the image processing was repeated. The magnitude and direction of the source displacement must be the same as used for the object under investigation. The phase change determined for the flat plate was subtracted from the measured phase change on the object under investigation, and scaled using the parameters given in Equation 2, to yield the object slope. The object slope was integrated to obtain the object shape.

\subsection{Surface strain measurement applying a correction for object slope and shape}

The displacement gradient components were corrected for the change in applied shear and the variation in the sensitivity vector across the object and are mapped onto the object surface, as detailed in Section 2.3. The surface strain components can be calculated by referring to the strain tensor, given in Equation 1. 


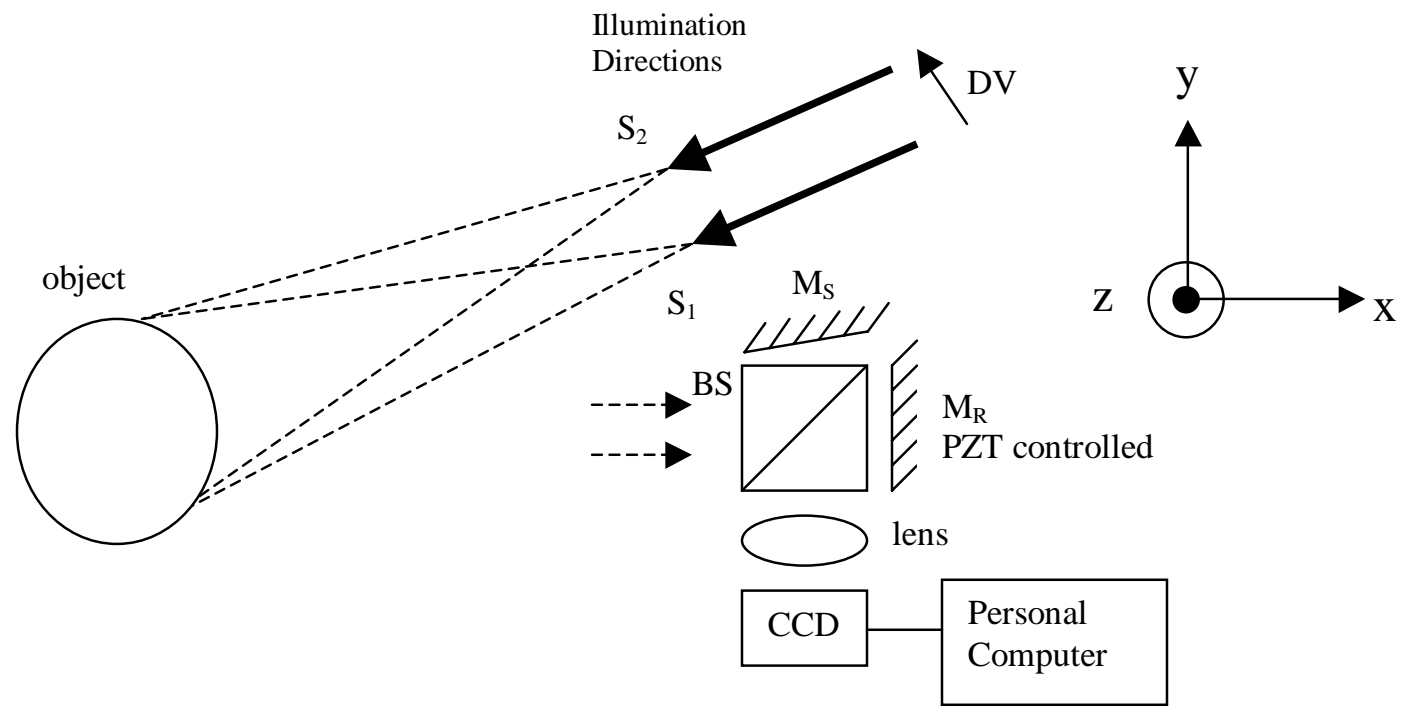

Figure 3 Experimental layout for slope and shape measurement. The source is displaced from $S_{1}$ to $S_{2}$ by a distance DV between camera frames. The shearing head consists of beamsplitter BS, shearing mirror $\mathrm{M}_{\mathrm{S}}$, reference mirror $\mathrm{M}_{\mathrm{R}}$, controlled by a piezoelectric transducer, a lens and a CCD camera.

\section{RESULTS}

The test object was a section of stainless steel gas main pipe of length $900 \mathrm{~mm}$ and diameter $90 \mathrm{~mm}$. This is shown in Figure 4. The pipe was constructed from two end plates and three pipe sections, joined by welds around the circumference of the pipe. The section of the pipe investigated using shearography contained a welded joint between a $15 \mathrm{~mm}$ and an $8 \mathrm{~mm}$ pipe section. The section of the pipe under investigation was coated with white paint.
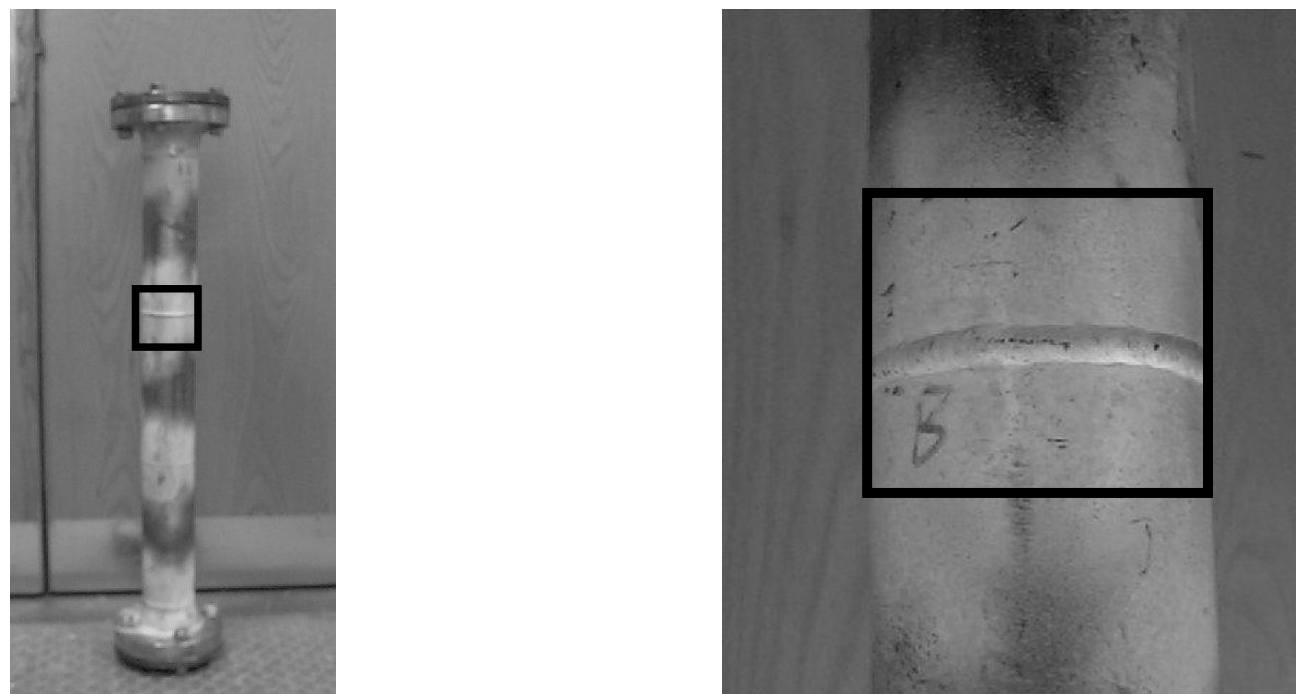

Figure 4 Photograph of (a) the gas main pipe and (b) a close-up of the section of pipe investigated using the Shearography system. The black border in both pictures indicates the field of view of the camera of approximately $90 \mathrm{~mm}$ by $90 \mathrm{~mm}$ 


\subsection{Multi-component surface displacement gradient measurement}

Displacement gradient measurements were performed using three illumination positions and two shear directions. The illumination positions were situated at $1 .(0.220 \mathrm{~m},-0.173 \mathrm{~m}, 0.270 \mathrm{~m}), 2 .(0.224 \mathrm{~m}, 0.176 \mathrm{~m}, 0.265 \mathrm{~m})$ and $3 .(-0.177$ $\mathrm{m}, 0.187 \mathrm{~m}, 0.210 \mathrm{~m})$. Shears of magnitude $10 \mathrm{~mm}$ were applied in the $\mathrm{x}$ and the $\mathrm{y}$ directions. This yielded six components of measured displacement gradient in total. The pipe was pressurised to $350 \mathrm{kPa}$ before recording the reference frames and depressurised to $0 \mathrm{kPa}$ before recording the phase-stepped signal frames. Figure 5 shows wrapped phase maps for the six measured displacement gradient components of a region of approximately $40 \mathrm{~mm}$ by $55 \mathrm{~mm}$. The intensity of scattered light from other regions of the surface within the field of view was insufficient to generate a wrapped phase map, when illuminated from one or more of the illumination directions.

After the unwrapping procedure six unwrapped phase maps of the measured displacement gradient components are obtained, these are shown in Figure 6.

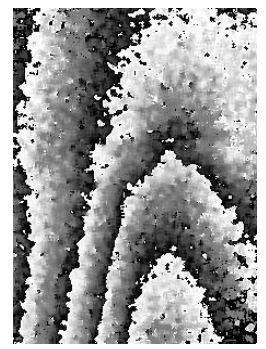

(a)

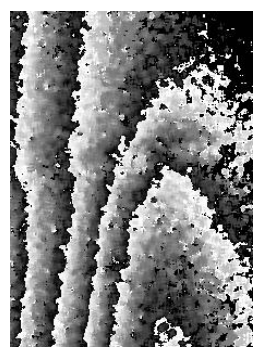

(b)

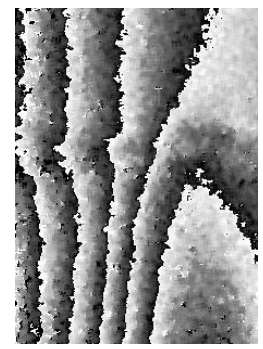

(c)

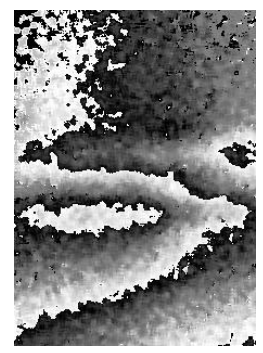

(d)

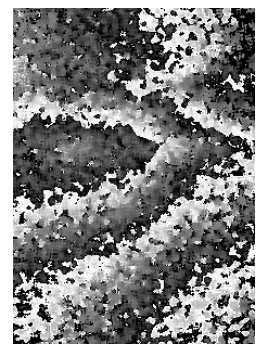

(e)

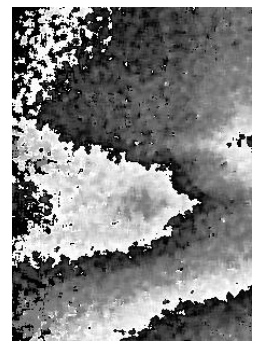

(f)

Figure 5 (a), (b) and (c) are respectively the wrapped phase maps of displacement gradient for applied shear in the $\mathrm{x}$ direction for illumination directions 1,2 and 3. (d), (e) and (f) are respectively the wrapped phase maps of displacement gradient for applied shear in the y direction for illumination directions 1, 2 and 3. In all pictures the weld is horizontally across the centre of the picture, where it is indicated by local changes in surface displacement gradient.

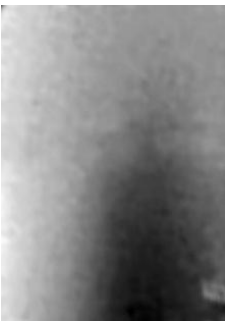

(a)

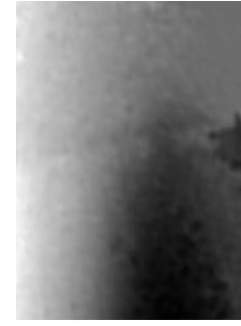

(b)

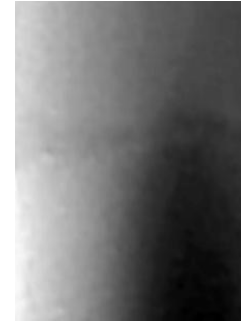

(c)

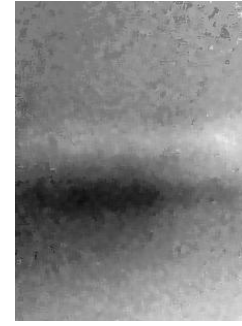

(d)

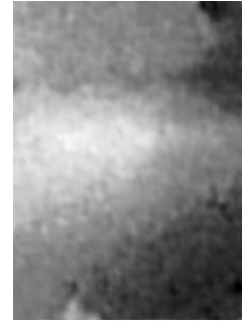

(e)

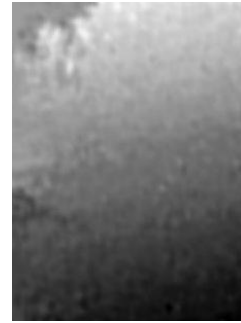

(f)

Figure 6 (a), (b) and (c) are respectively the unwrapped phase maps of displacement gradient for applied shear in the $\mathrm{x}$ direction for illumination directions 1, 2 and 3. (d), (e) and (f) are respectively the unwrapped phase maps of displacement gradient for applied shear in the y direction for illumination directions 1,2 and 3. The white regions represent regions of maximum displacement gradient. In all pictures the weld is horizontally across the centre of the picture, where it is indicated by local changes in surface displacement gradient.

\subsection{Slope and shape measurement}

The object surface slope and shape were measured using the source displacement technique described in Section 3.2. For these measurements, channel 3 of the multi-component shearography system was used, with the source position initially at $(-0.177 \mathrm{~m}, 0.187 \mathrm{~m}, 0.210 \mathrm{~m})$. The slope was measured using an applied shear of $10 \mathrm{~mm}$ in the $\mathrm{x}$ direction and a source displacement of $300 \mu \mathrm{m}$ in the DV direction. A measurement of the phase change across a flat plate test object was also performed for a source displacement of $300 \mu \mathrm{m}$ in the DV direction. This phase change was subtracted from the phase change on the pipe to obtain the surface slope, in the shear direction, for the pipe. A value for the 
constant $\mathrm{K}$ of $11 \mathrm{~m}^{-1}$ was calculated numerically and this was used to perform the scaling of the measured slope. The surface slope was integrated to recover the object shape. Figure 7 shows the wrapped slope fringes from the pipe and the flat plate. Figure 8 shows the recovered object shape without and with the correction for the phase change across the flat plate.

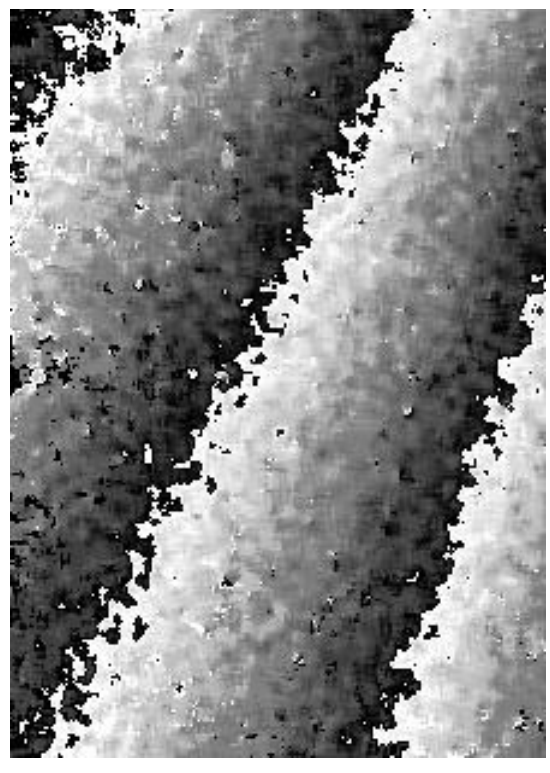

(a)

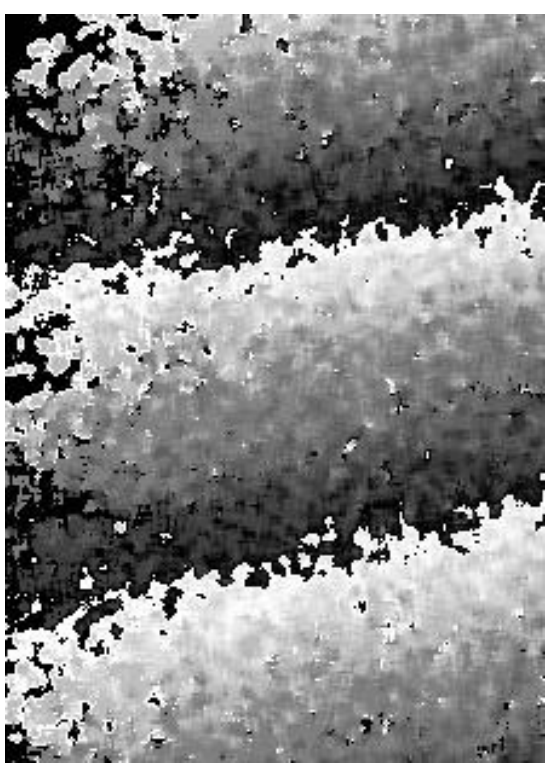

(b)

Figure 7 shows wrapped phase maps, of a region of approximately $40 \mathrm{~mm}$ by $55 \mathrm{~mm}$, (a) the measured slope in the $\mathrm{x}$ direction of the pipe and (b) the phase change on the flat plate for applied shear in the $\mathrm{x}$ direction.

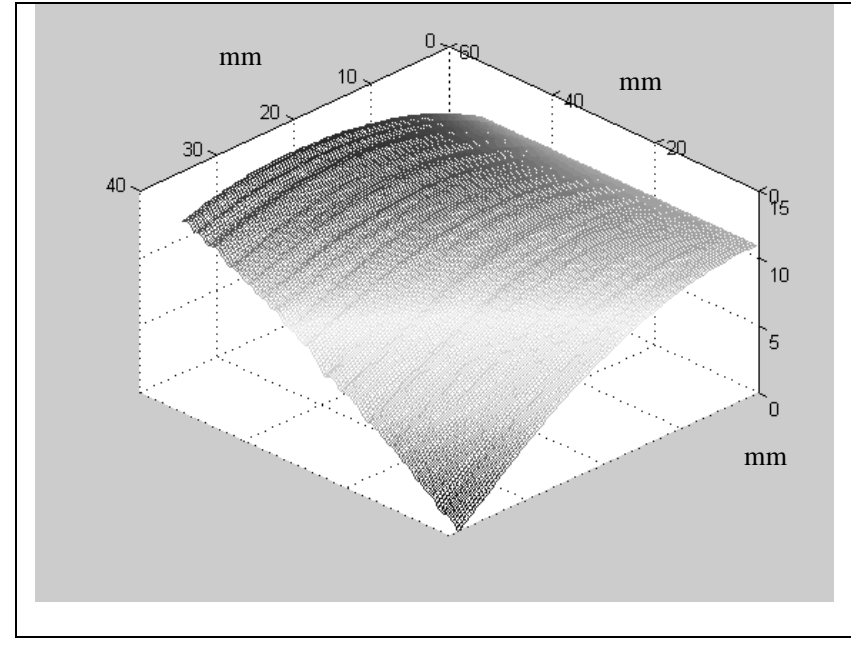

(a)

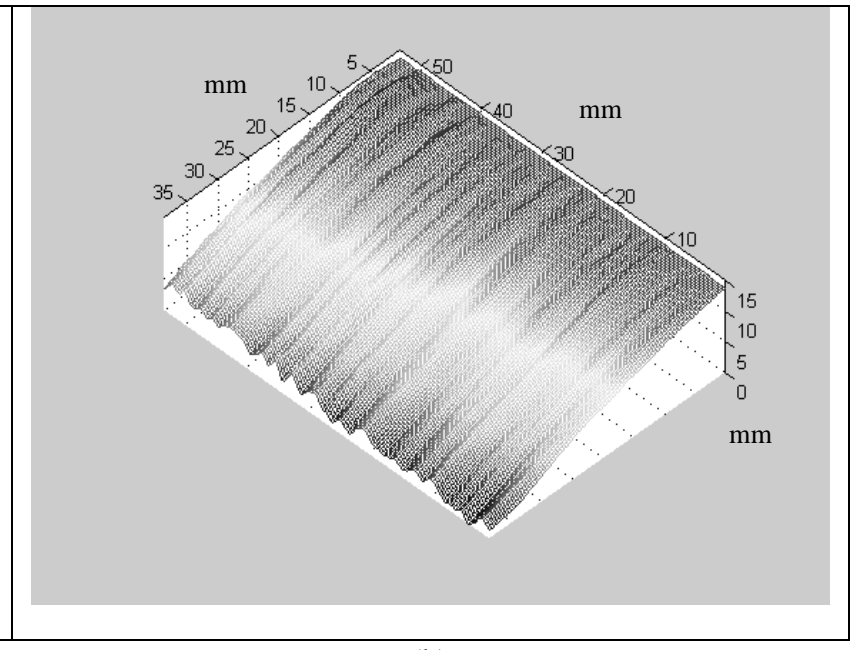

(b)

Figure 8 shows the object shape obtained (a) without performing the correction for the phase change across the flat plate and (b) performing the correction for the phase change across the flat plate. 


\subsection{Surface strain measurement applying a correction for object slope and shape}

The measured displacement gradient components are corrected using the object slope and shape information and mapped onto the object surface. Figure 9 shows the six components of displacement gradient relative to the local object surface. The range of the data is $-32 \mu \varepsilon$ (black) to $+32 \mu \varepsilon$ (white). The images are of a region approximately $40 \mathrm{~mm}$ by $55 \mathrm{~mm}$ in size.

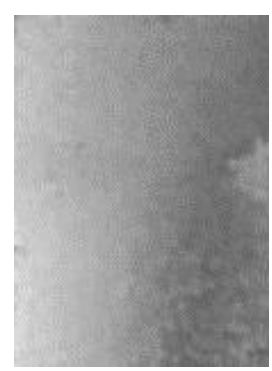

(a)

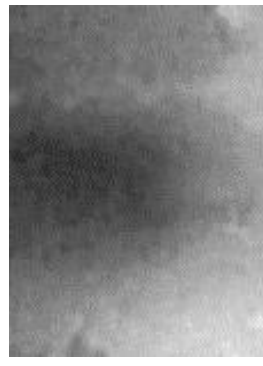

(d)

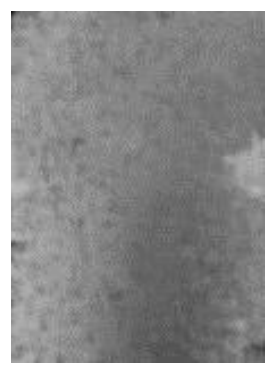

(b)

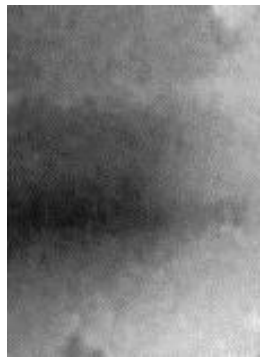

(e)

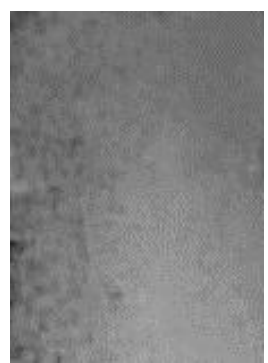

(c)

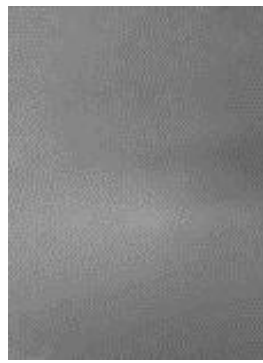

(f)

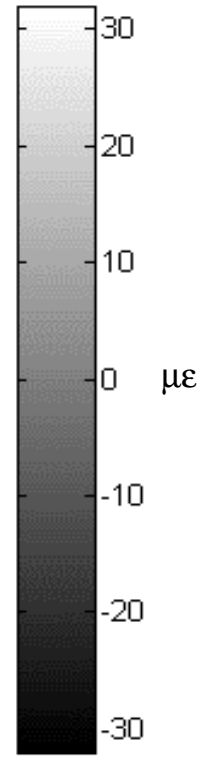

(g)

Figure 9 shows the six displacement gradients mapped relative to the local surface profile. These are (a) du/dx, (b) dv/dx, (c) dw/dx, (d) du/dy, (e) dv/dy and (f) dw/dy. (g) is the greyscale in micro strain. The images are of a region approximately $40 \mathrm{~mm}$ by $55 \mathrm{~mm}$ in size. The range of the data is $-32 \mu \varepsilon$ (black) to $+32 \mu \varepsilon$ (white) with a resolution of $1 \mu \varepsilon$.

\section{DISCUSSION}

A theoretical calculation of the hoop strain and longitudinal strain for a pressurised pipe were made and the values obtained were comparable with the in-plane displacement gradient components measured using the shearography system.

In this paper errors in the measurement of displacement gradient, due to the surface slope and shape are investigated and corrected. For a curved object, or for an object tilted relative to the plane of the camera image, the applied shear measured in the plane of the camera image is smaller than the actual applied shear at the object surface. For the slopes that can practically be measured by this shearography system, between -1 and +1 , the actual applied shear is larger than the measured applied shear by a maximum of approximately $40 \%$. There is also an error due to the direction of the sensitivity vector varying across the object surface. A knowledge of the object shape is used to correct for this variation in the sensitivity vector across the object surface.

A source displacement generates a phase change across a flat plate surface, where the surface slope is zero. The surface slope measurements are corrected for this phase change. When the surface slope and shape determination are performed the geometry of the illumination and imaging positions determines the sensitivity of the correlation fringes to surface 
slope in a complex way. In this paper a slope sensitivity constant, $\mathrm{K}$, was used to perform the scaling of the measured slope. This constant was calculated numerically using a model of the fringe formation.

For both the displacement gradient and the slope measurements the slope of the surface relative to the illumination and imaging directions limits the regions on the pipe where satisfactory reading may be taken. This is because sufficient optical power is required at the camera to generate a satisfactory wrapped phase map. It may be possible to change the surface coating or increase the laser power to perform measurements over a larger region of the surface of the pipe.

\section{CONCLUSIONS}

Shearography has successfully been used to measure the six displacement gradient components, the object slope in two directions and the object shape in the region of a welded joint in a gas main pipe. The slope and shape information has been used to correct the displacement gradient measurements, with a correction made of up $40 \%$ to the displacement gradient. This was achieved using the existing Cranfield multi-component shearography system, with the addition of a translation stage which was fitted to one of the existing source positions to provide the surface slope and shape measurement capability. The displacement gradient was mapped onto the object surface to obtain in-plane and out-ofplane displacement gradient components relative to the local profile of the object surface.

\section{ACKNOWLEDGEMENTS}

This work was partially supported by the European Union under Brite Euram project No. BE96 3116, MuWaS, Multiple Wavelength Shearography and by the Engineering and Physical Sciences Research Council, UK.

\section{REFERENCES}

1. J. A. Leendertz and J. N. Butters, "An image-shearing speckle pattern interferometer for measuring bending moments", J. Phy. E. 6, pp. 1107-1110, 1973.

2. Y. Y. Hung, "Shearography: a new optical method for strain measurement and nondestructive testing", Opt. Eng. 21 (3), pp. 391-395, 1982.

3. K. Creath, "Temporal phase measurement methods" in "Interferogram analysis, digital fringe measurement techniques", ed. D. W. Robinson and G. T. Reid, 1993 (Bristol: Institute of Physics).

4. J-R Huang, H. D. Ford and R. P. Tatam, "Slope measurement by two wavelength electronic shearography", Opt. Laser. Eng., 27, pp. 321-333, 1997.

5. C. J. Tay, F. S. Chau, H. M. Shang, V. P. W. Shim and S. L. Toh, "The measurement of slope using shearography", Opt. Laser.Eng. 14, pp. 13-24, 1991.

6. R. M. Groves, S. W. James and R. P. Tatam, "Shape measurement by source displacement in threedimensional shearography", Proc. SPIE 4101, pp. 121-129, 2000.

7. S. W. James and R. P. Tatam, "Time-division-multiplexed 3D shearography”, Proc. SPIE 3744, pp. 394-403, 1999.

8. J. Takezaki and Y. Y. Hung, "Direct measurement of flexural strains in plates by shearography", J. Appl. Mech. 53, pp. 125-129, 1986. 